\title{
Die Zukunft kommt langsam
}

\author{
Primärversorgungszentren (PVZ) sollen Versorgungslücken am Land stopfen \\ und Ambulanzen entlasten. Minister Wolfgang Mückstein will den schleppenden \\ Ausbau der PVZ mit 100 EU-Millionen wieder auf Spur bringen. \\ Von Josef Ruhaltinger
}

Bevor Wolfgang Mückstein Minister wurde und noch über kein Presseteam verfügte, hatte der Allgemeinmediziner für Fachjournalisten jederzeit ein offenes Ohr. Es war im Frühjahr 2019 - Pandemie und Ministeramt waren für Mückstein noch irrationale Visionen -, als der Partner des ersten Wiener Primärversorgungszentrums Medizin Mariahilf binnen 48 Stunden Zeit für ein Interview hatte - und das an einem frühen Vormittag. Auf die Flexibilität angesprochen lieferte der heutige Minister eine einfache Erklärung: „Ich habe mich mit meinen zwei Kollegen abgestimmt." Und er wusste um den Hintergrund der Journalistenfrage: „In einer Einzelordination müsste ich jetzt in der Sprechstunde stehen. " Damit beschrieb Wolfgang Mückstein einen aus ärztlicher Sicht wesentlichen Vorteil seiner Partnerschaft in einem Primärversorgungszentrum: „Wenn ein Partner verhindert

ist, übernimmt ein Kollege den Dienst. Und ich revanchiere mich später." Verbesserte Work Life-Balance für die agierenden Ärzte und Kontinuität in der Betreuung der Patienten - vereinfacht sind dies die großen Assets eines Primärversorgungszentrums.

\section{Neues Verständnis von Gesundheitsversorgung}

Wolfgang Mückstein gilt als einer der Vorreiter des Primary Health-Gedankens in Österreich. Gemeinsam mit Franz Mayerhofer, dem ursprünglichen Praxisinhaber in Mariahilf, gründete er - mit Fabienne Lamel als dritter Partnerin - 2015 Österreichs erstes Primärversorgungszentrum. Mit seiner Berufung zum Minister für Gesundheit verbanden sich viele Hoffnungen in der Primary Health-Community. Den zuletzt war die Umsetzung der Pläne für ein Primärversorgungssystem in Österreich gehörig ins Stocken gekommen. 2017 hatte das Gesundheitsreformumsetzungsgesetz von 75 Primärversorgungseinheiten (PVE) bis 2021 fabuliert. Laut Gesundheitsministerium waren es Stand Juli 2021 genau 28, die ihre Arbeit aufgenommen haben. Außer etlichen kollegialen Besuchen in diversen Primärversorgungseinrichtungen war bisher im PVE-Bereich von einem Mückstein-Boost nichts zu spüren. Stefan Korsatko, Sprecher des

Förderungen. ForumsSprecher und PVZ-Arzt Stefan Korsatko hofft auf Mittel für 60 weitere Gründungen und 90 weitere Projekte aus dem PV-Bereich.
Österreichischen Forums für Primärversorgung und ärztliches Teammitglied des Grazer Primärversorgungszentrums „Medius“, zeigt sich mit dem Erreichten zufrieden: „Die Idee ist ins Rollen gekommen und unumkehrbar.“ Aber er weiß: „75 Zentren werden es in nächster Zeit nicht so schnell.“ Das Gesundheitsministerium verweist in einem Mail auf die "Covid-19-Pandemie, durch die die Umsetzung von PVE in Österreich langsamer fortgeschritten ist als ursprünglich geplant." Eine Sprecherin des Ministers versichert dabei: „In den kommenden Jahren soll es mehr Dynamik in diesem Bereich geben."

\section{Ein Fonds schürt Hoffnungen}

Stefan Korsatko ist für die mittelbare Zukunft des Primary Health Care-Systems in Österreich zuversichtlich. Sein Optimismus hat einen speziellen Grund: den österreichischen Aufbauund Resilienzplan (ARP). Er ist Teil des europäischen Aufbauinstruments „Next Generation EU“, das mithilfe von 750 Mrd. Euro Europa aus der Pandemie-Agonie holen soll. Mit dessen Herzstück, der „Aufbau- und Resilienzfazilität“, werden den Mitgliedstaaten anteilsmäßig insgesamt 672,5 Mrd. Euro an Zuschüssen 


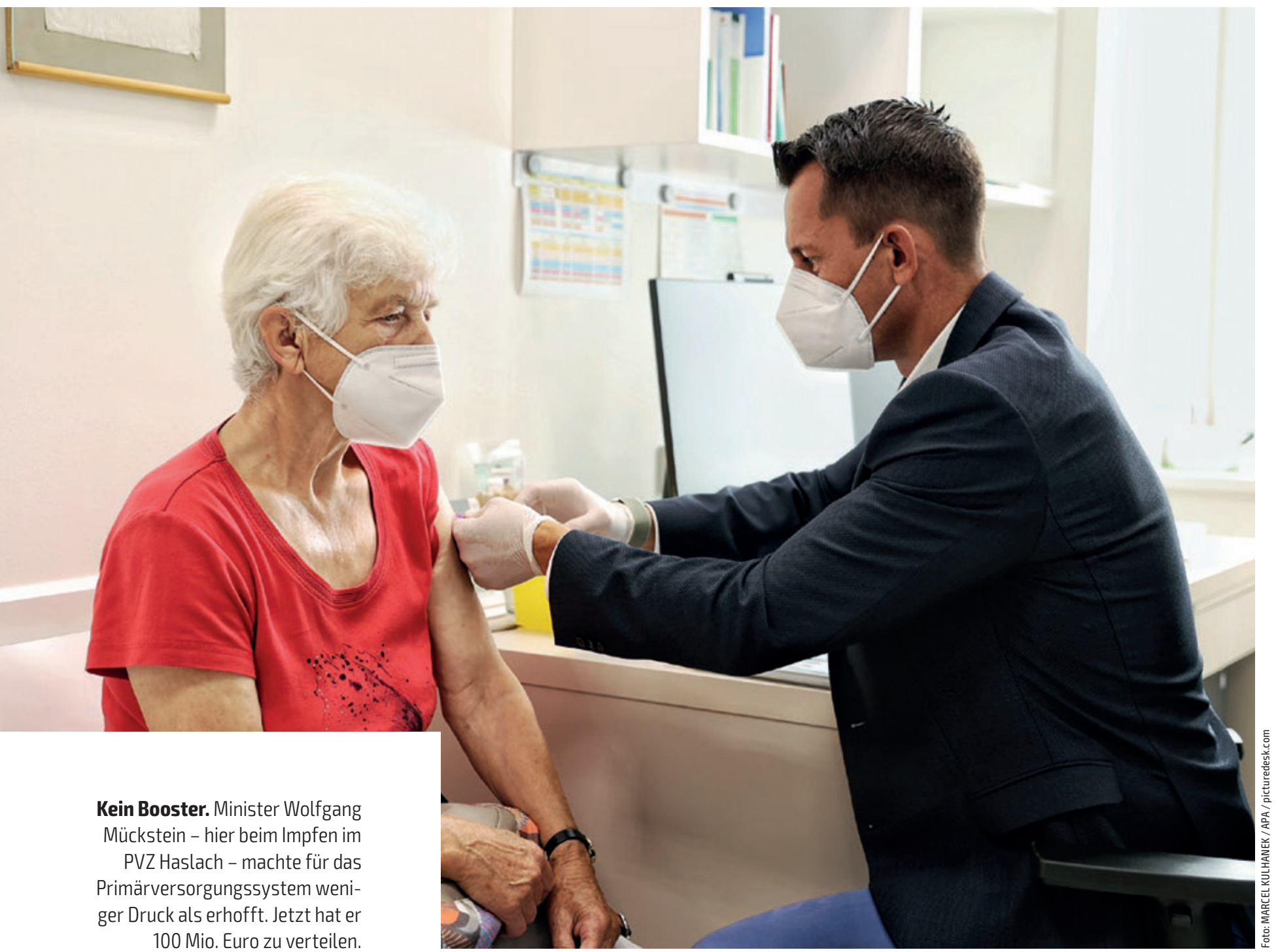

und Darlehen zur Verfügung gestellt. Nach positiver Begutachtung des nationalen Investitionsplans durch die EU-Kommission am 21. Juni - das Geld muss zweckgewidmet in den digitalen Aufbau und klimaschützende Maßnahmen fließen - kann Österreich mit mindestens drei Mrd. Euro für nationale Zukunftsinvestitionen rechnen.

Ein Stück davon darf der Bereich der Primary Health Care für sich in Anspruch nehmen: Im Kapital 4a Gesundheit des ARP sind 100 Millionen Euro für die Attraktivierung des Bereichs Primärversorgung vorgesehen. Aktuell wird über die genaue Mittelverwendung zwischen Ministerium, Ärztekammer und Kassen verhandelt. Die Presseabteilung des Gesundeitsministeriums schreibt auf Anfrage, dass mit dem Geld „Maßnahmen zur Attraktivierung der Allgemeinmedizin sowie der gesamten Primärversorgung vor allem im ländlichen Raum" getroffen werden sollen. Dazu zählten der „flächendeckende Roll out“ von Primärversorgungszentren und -netzwerken“ sowie der „Ausbau der digitalen Infrastruktur sowie die Steigerung der Energieeffizienz in den Praxen“. Speziell gefördert werden sollen auch „die Vernetzung der unterschiedlichen Gesundheits- und Sozialberufe sowie die Angebote zur Fort- und Weiterbildung der interprofes- sionellen Teams“. Was aber genau kommt, steht nicht fest. Stefan Korsatko verfolgt die Gespräche aus nächster Nähe: „Es ist die Rede von 60 weiteren Gründungen von PV-Einheiten sowie von 90 weiteren verschiedenen Projekten aus dem Bereich der Primärversorgung. " Dabei soll es um verlorene Zuschüsse in Höhe von 50 Prozent (nicht rückzahlbar) der Investitionssummen gehen. Administriert werden die 100 Mio. Euro von der Gesundheit Österreich GmbH.

\section{Alternative zu 5-Minuten-Medizin}

Primärversorgungseinheiten PVE sind die Chance, der HausarztMedizin jene Kompetenzen zur Seite zu stellen, die eine nachhaltige und umfassende Gesundheitsversorgung erleichtern. Dabei geht es nicht nur darum, die Ambulanzen zu leeren, sondern den Ursachen von Krankheit umfassender auf den Grund zu gehen. Die flankierenden Gesundheitsangebote eines Primärversorgungszentrums sorgen für mehr Kontinuität in Therapie und Prävention. Sie sind das Herzstück des Primärversorgungsgedankens. Wolfgang Hocke, Initiator und Dynamo des federführenden PVZ Enns, hob in einem früheren Gespräche mit der ÖKZ vor allem den Teamgedanken hervor: „In unserer Gemeinschaft aus 
Ärzten und anderen Gesundheitsdienstleistern lässt sich mehr bewegen, als ein einzelner Allgemeinmediziner bei aller Selbstaufopferung erreichen kann. " Ein Patient, der die Physiotherapeutin, den Sozialarbeiter oder die Ernährungsberaterin des Primärversorgungszentrums besucht, bleibt in Reichweite des Hauses und des behandelnden Arztes.

\section{Neue Form der Versorgung}

Primärversorgungszentren sind in den Augen von immer mehr Allgemeinmedizinern die Chance, die niedergelassene Gesundheitsversorgung weiterzuentwickeln. Denn eines ist bei aller notwendigen Kritik klar. Existenzängste unter konventionellen AllgemeinmedizinerInnen sind unbegründet. Die PVE können den knapp 4.000 allgemeinmedizinischen Ordinationen betriebswirtschaftlich nicht gefährlich werden - eine Angst, die in den Standesvertretungen immer wieder unter der Hand geäußert wird. Stefan Korsatko spricht daher auch „von notwendigen Ergänzungen durch die PVZ, keinesfalls ein Ersatz für das extramurale System“. Die PVE können neue Formen der Gesundheitsversorgung in eine sich verändernde Gesellschaft implantieren. Teamarbeit wird wichtiger.

Das Gesundheitsreformumsetzungsgesetz (GRUG) schuf 2017 die erforderlichen rechtlichen Rahmenbedingungen zur Errichtung von Primärversorgungseinheiten. Und mit dem Bundesrahmenvertrag vom 2. April 2019 wurden die Details geregelt: PVE sollen sich aus Patientensicht durch gute Erreichbarkeit, längere Öffnungszeiten und ein umfassenderes Leistungsangebot auszeichnen. Die genaue Ausgestaltung der Einrichtungen variiert

dem „Erweiterten Teams“, die hinzugezogen werden „können“, in der Realität aber das Um- und Auf des Primärversorgungsgedankens repräsentieren. Infrage kommen Physiotherapeuten, Diätologen, Ergotherapeuten, Psychologen, Psychotherapeuten, Hebammen bis hin zu Sozialarbeitern. Die meist angestellten Gesundheitsberufe sorgen für die nachhaltige Betreuung der Patienten. Auch soll ein Ordinationsmanager Organisatorisches und Bürokratisches übernehmen können. Leiter einer PVE sind aber immer Ärzte.

Der „Nurse“ kommt in einer PVE besonderer Stellenwert zu: Sie übernimmt die erste Lotsenfunktion bei Neupatienten, wer innerhalb des PVE-Teams dem Patienten am besten helfen kann. Stammpatienten wird immer ihr „Hausarzt“ zugeteilt. Die NurseFunktion ist für ein funktionierendes Zentrum essenziell.

\section{Föderalismus am Werken}

Das Thema der Primärversorgung erfreut sich in den neun Bundesländern völlig unterschiedlicher Beliebtheit. Dem Hang zum österreichischen Gesundheitsföderalismus entsprechend hängt die Näherung in erster Linie von den handelnden Personen ab. Die Haltung der Entscheidungsträger in den jeweiligen Gebietskrankenkassen, Länderärztekammern und unter den Ärztinnen und Ärzten reicht von glühenden Verfechtern eines vielschichtigen Behandlungsbegriffes bis hin zu Totalverweigerern, die in den PVZ den Abgesang auf den traditionellen Hausarztberuf sehen. Franz Kiesl war maßgeblich daran beteiligt, dass Oberösterreich im Bereich der Primärversorgung eine Vorreiterrolle einnimmt. Kiesl war in der OÖGKK für PVE verantwortlicher Direktor und ist seit 1.1.2020 Leiter des Bereichs Versorgungsmanagement 1 in der Österreichischen Gesundheitskasse und dort wieder für PVE zuständig: „Uns fehlt es an Nachwuchs in der Allgemeinmedizin, insbesondere auch deshalb, weil viele Studierende ein falsches Bild vom Beruf der Hausärztin bzw. des Hausarztes haben“, beschreibt Kiesl in einem Interview mit PVE.gv.at (offizielle Website des Ministeriums zum Thema Primärversorgung) das Problem des fehlenden Nachwuchses bei Hausärzten. „Wir stecken mitten in einem Generationenwechsel. Viele Jungärztinnen und Jungärzte haben einen anderen Lifestyle, ein geändertes Rollenbild. Das führt zu lokalen und temporären Nachbesetzungsproblemen." PVE bieten

von Bundesland zu Bundesland. Laut Grundlagenvertrag besteht das Kernteam einer PV-Einrichtung aus mindestens drei AllgemeinmedizinerInnen, einer diplomierten Gesundheits- und KrankenpflegerIn sowie einer Ordinationsassistentin. Fakultativ kann zusätzlich auch eine KinderärztIn Teil des Kernteams werden. Als Mindestöffnungszeit ist bei drei Ärztestellen ein Korridor von mindestens 40 bis 50 Sprechstunden ganzjährig in der Zeit von 7 bis $19 \mathrm{Uhr}$ vorgesehen. Sind es mehr Ärzte, verlängern sich die Öffnungszeiten. Der Rahmenplan spricht außerdem von
Hausärzten planbare Arbeitszeiten - ein Luxus, den traditionelle Gemeindeärzte am Land nicht kennen. Die deutlich verbesserte Work Life-Balance soll den Beruf des Allgemeinmediziners attraktiver machen und helfen, den drohenden Versorgungslücken am Land vorzubeugen. Und im urbanen Raum wirken PVE als Puffer für Spitalsambulanzen - und dies nicht zuletzt wegen der Öffnungszeiten. „Medizin Mariahilf“ hat 50 Stunden pro Woche und 52 Wochen im Jahr geöffnet - ein Angebot, das Einzelkämpfer nicht erbringen können. 